\title{
DE LOS PAQUETES DIDÁCTICOS HACIA UN REPOSITORIO DE OBJETOS DE APRENDIZAJE: UN RETO EDUCATIVO EN MATEMÁTICAS. USO DE LAS GRÁFICAS, UN EJEMPLO ${ }^{1}$
}

(FROM MATHEMATICS DIDACTIC PACKAGES TOWARDS A LEARNING OBJECTS REPOSITORY:
AN EDUCATIVE CHALLENGE IN MATHEMATICS. AN EXAMPLE: GRAPHICS USE)

Liliana Suárez Téllez

Francisco Cordero Osorio

Patricio Daowz Ruiz

Pedro Ortega Cuenca

Alfonso Ramírez Ortega

José Luis Torres Guerrero

Instituto Politécnico Nacional, (México)

\section{RESUMEN}

En este estudio se muestran los principales aspectos del diseño de los Paquetes Didácticos de Matemáticas (PDM). Gracias a su diseño, los PDM pueden transformarse en repositorios de objetos de aprendizaje de acceso libre para cualquier discente interesado. En su descripción se pone énfasis en la reutilización de las actividades de aprendizaje y en la concepción de un diseño didáctico robusto y flexible que permita recorrer diversas trayectorias de aprendizaje, mediante redes de actividades que contribuyan a lograr objetivos educativos complejos. Los PDM integran los conocimientos de la investigación en Matemática Educativa con la innovación de las TIC.

\begin{abstract}
This work points out the main aspects from the design of Mathematics Didactic Package (MDP). Due to their didactic well designed aspects, they can be turn into Learning Objects Repositories, which could be accessed by any interested learner. A main emphasis is made on the reusability of their learning tasks. That is to say, the MDP can be used as a starting point in the creation of a didactic flexible design to build up learning sequences such as learning networks with more complex learning objectives. Finally, it is important to mention that the MDP integrate the products of the research in Mathematics Education field and the use of the ICT.
\end{abstract}


Liliana Suárez, Francisco Cordero, Patricio Daowz, Pedro Ortega, Alfonso Ramírez y José Torres

DE LOS PAQUETES DIDÁCTICOS HACIA UN REPOSITORIO DE OBJETOS DE APRENDIZAJE:

UN RETO EDUCATIVO EN MATEMÁTICAS. USO DE LAS GRÁFICAS, UN EJEMPLO

\section{INTRODUCCIÓN}

El Proyecto 'Paquetes Didácticos para los cursos de Matemáticas' (PDM), coordinado por el actual Centro de Tecnología Educativa del Instituto Politécnico Nacional desde su creación en 2001, tiene como propósito dotar al profesor y al estudiante de materiales de calidad, elaborados usando el conocimiento generado por las investigaciones y aplicado de manera sistemática, que les permitan trabajar conjuntamente para lograr los objetivos institucionales del área de matemáticas. Los PDM se han desarrollado para el aprendizaje de las matemáticas en el Nivel Medio Superior del Instituto Politécnico Nacional (IPN). El paquete didáctico es un conjunto de materiales que concretan operativamente los cuatro organizadores del currículo: objetivos, contenidos, metodología y evaluación. En particular, las estrategias didácticas y metodológicas, los conocimientos matemáticos y los elementos teóricos para ampliar la cultura matemática de los estudiantes.

Para el diseño del paquete se consideran el marco institucional y algunos estándares, tanto nacionales como internacionales y se define una gama de experiencias de aprendizaje congruente con las competencias que ahí se establecen. Los materiales necesarios para lograr los ambiciosos, pero pertinentes, objetivos de la educación actual son complejos y requieren de un profesor con una cultura profesional, capaz de aprovechar creativamente el sustento técnico que proporciona el conocimiento profesional, principalmente el que proviene de los resultados de la investigación en educación matemática. Una parte fundamental del proyecto corresponde, entonces, a la familiarización y capacitación del profesor en el manejo del paquete.

Con este proyecto se tiene una oportunidad para cumplir con los objetivos institucionales pero estamos todavía lejos de contar con indicadores válidos y confiables en algunos de los aspectos fundamentales, señaladamente el aprendizaje de los estudiantes y del desempeño docente. Sin embargo, con estos proyectos se sientan las bases para avanzar en soluciones de fondo a estos aspectos tan descuidados y en los que actualmente se tienen indicadores inadecuados porque dejan de lado la verdadera piedra de toque de la práctica docente: qué puede hacer un alumno con sus conocimientos, qué puede demostrar que sabe cuando se evalúa su desarrollo en términos de los objetivos de la institución.

El uso de los resultados de la investigación y la integración de las Tecnologías de la Información y la Comunicación mencionadas ha permitido un mejor aprovechamiento por parte de los estudiantes, así como también ha sentado los 
Liliana Suárez, Francisco Cordero, Patricio Daowz, Pedro Ortega, Alfonso Ramírez y José Torres

DE LOS PAQUETES DIDÁCTICOS HACIA UN REPOSITORIO DE OBJETOS DE APRENDIZAJE:

UN RETO EDUCATIVO EN MATEMÁTICAS. USO DE LAS GRÁFICAS, UN EJEMPLO

primeros pasos para que profesores y estudiantes del IPN conformen una Comunidad Virtual de Aprendizaje (AIM-NMS-IPN, 2006). En Suárez et al (2005) se describen los principales elementos de estos materiales que incluyen las herramientas tradicionales así como herramientas innovadoras como discos compactos, sitios en Internet y Ambientes Virtual de Aprendizaje administrados mediante la plataforma Blackboard.

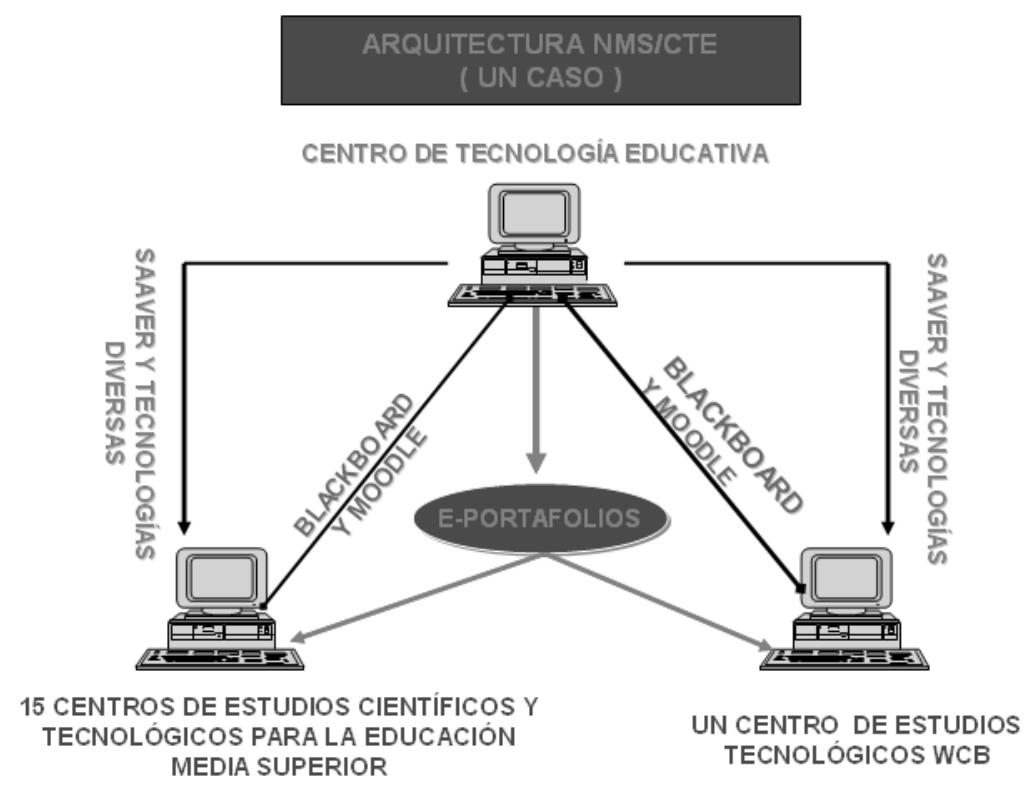

Fig. 1. Incorporación de Tecnologías Educativas en el Nivel Medio Superior.

Es de interés para este proyecto mostrar como la concepción y constitución de los Paquetes Didácticos de Matemáticas reúnen las características de reusabilidad de los materiales curriculares basados en las redes. El propósito de esta tarea es mostrar una metodología de construcción de contenidos digitales en la que se incorporen profesores y estudiantes en el desarrollo de contenidos educativos digitales en el Nivel Medio Superior del Instituto Politécnico Nacional.

Cada Paquete Didáctico comprende: Libro para el estudiante (Véase IPN, 2004), Libro para el profesor (Véase IPN, 2006), Disco para el estudiante, Disco 
Liliana Suárez, Francisco Cordero, Patricio Daowz, Pedro Ortega, Alfonso Ramírez y José Torres

DE LOS PAQUETES DIDÁCTICOS HACIA UN REPOSITORIO DE OBJETOS DE APRENDIZAJE:

UN RETO EDUCATIVO EN MATEMÁTICAS. USO DE LAS GRÁFICAS, UN EJEMPLO

para el profesor, Sitios en Internet (Véase AIM-NMS-IPN, 2005), Ambientes para la capacitación en el manejo de los paquetes didácticos y Espacios de trabajo para las comunidades que realizan el seguimiento y la evaluación (Véase AIM-NMS-IPN, 2002).

\section{Secuencias de Actividades de Aprendizaje.}

Los Paquetes Didácticos de Matemáticas comprenden un amplio abanico de actividades de aprendizaje: problemas, problemas con guía, proyectos, lecturas, ejercicios, tareas y autoevaluaciones. Esta variedad de actividades es necesaria para el cumplimiento de los objetivos del programa del curso y de la dimensión matemática de las Competencias básicas del estudiante de bachillerato (Documentos de la Reforma Académica. IPN, 2004)

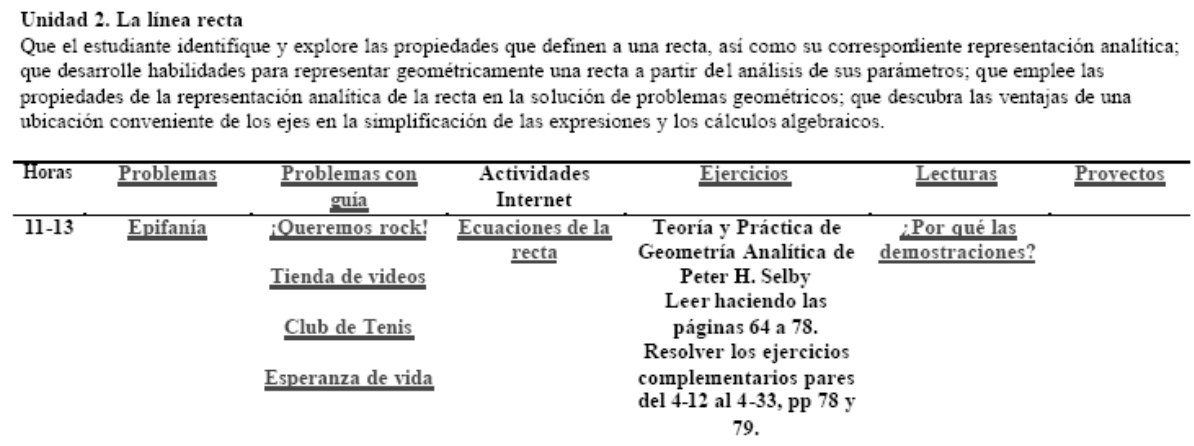

Fig. 2. Secuencia de actividades para estudiar la línea recta.

Las competencias básicas se refieren al dominio, por parte del estudiante, de los conocimientos, habilidades, valores y actitudes que son indispensables tanto para la comprensión del discurso de las ciencias, las humanidades y la tecnología, como para su aplicación en la solución de los problemas de su vida escolar, laboral o cotidiana, por lo que son -o deben ser- comunes todos los bachilleratos del país. Para fomentar estas competencias, no es suficiente un único tipo de actividad de aprendizaje, se requiere el uso articulado de las actividades propuestas en los Paquetes Didácticos de Matemáticas, integración, las conexiones y las aplicaciones de los conocimientos tanto dentro como fuera de las Matemáticas. 
Liliana SuÁrez, Francisco Cordero, Patricio Daowz, Pedro Ortega, Alfonso Ramírez y José Torres

DE LOS PAQUETES DIDÁCTICOS HACIA UN REPOSITORIO DE OBJETOS DE APRENDIZAJE:

UN RETO EDUCATIVO EN MATEMÁTICAS. USO DE LAS GRÁFICAS, UN EJEMPLO

A continuación proporcionamos comentarios sobre la importancia de cada una de estas actividades.

Problemas: Aquí se presenta un enunciado en el que se pide la respuesta a una o más preguntas y al alumno le corresponde responder. El profesor únicamente orienta el trabajo del alumno, sin ser él quien resuelva lo que se pide. La idea es que cada estudiante se vaya acostumbrando a tomar decisiones y a justificarlas. Para ello debe comenzar por una lectura cuidadosa del texto, encontrarle un sentido a la situación planteada, establecer una forma de representar la situación mediante una tabla, gráfica o expresión algebraica (mejor si utiliza las tres) y al trabajar con ellas, podrá responder lo que se le pide. Pero no termina aquí su trabajo. Debe darse cuenta por sí mismo si su respuesta tiene sentido, es decir si es aceptable a partir de la situación presentada en el enunciado. Como es una actividad de aprendizaje, encontrar una respuesta a la situación planteada no concluye el problema, éste continúa y se amplía al buscar otras formas de resolverlo o el establecimiento de un método de solución, que facilite el tratamiento de otras situaciones similares y el planteamiento de otras preguntas.

En los problemas con guía se tienen sesiones similares a la de los problemas. La diferencia consiste en que, en los problemas con guía, el enunciado incluye actividades que los alumnos pueden realizar para responder la pregunta (o preguntas) principales del problema. De esta manera se dirige al alumno hacia un camino o forma de resolver el problema. Una de las intenciones de estas orientaciones es disminuir la parálisis, tensión y angustia que algunos alumnos pueden experimentar con los problemas que dejan abierta la vía de solución.

Los proyectos son problemas que requieren de mayor tiempo para trabajarlos y se desarrollan principalmente fuera del salón de clases. La intención es fomentar la importancia de la perseverancia en el trabajo y de cumplir con los compromisos que se hacen. Para que un equipo pueda entregar un buen reporte de un proyecto se requiere que se interesen en él, que no lo vean como una tarea más que se deja en una materia para la cual basta entregar un reporte donde se anote lo que el alumno sabe que quiere el profesor.

Las lecturas podrían verse como simple complemento al curso, una introducción de temas que predispongan al alumno para el trabajo en serio de la materia. Pero no es así. El alumno debe desarrollar su habilidad de leer, de manera que pueda aprender de ellas. Se espera que las lecturas propuestas sean puntos de partida para discutir los temas que tratan. Para que esto se logre, se requiere la elaboración previa de un 
Liliana Suárez, Francisco Cordero, Patricio Daowz, Pedro Ortega, Alfonso Ramírez y José Torres

DE LOS PAQUETES DIDÁCTICOS HACIA UN REPOSITORIO DE OBJETOS DE APRENDIZAJE:

UN RETO EDUCATIVO EN MATEMÁTICAS. USO DE LAS GRÁFICAS, UN EJEMPLO

cuestionario que el profesor debe tener y el cual sirva de guía para la discusión de la lectura. Fomentar en el alumno una lectura crítica y reflexiva (cuidadosa) contribuye para la elaboración de argumentos mejor estructurados, y una comunicación más eficaz de sus ideas. Esto es particularmente importante porque estamos inmersos en información de todo tipo, y se requieren habilidades para hacer a un lado la información que no es relevante y, en cambio, analizar cuidadosamente la que sí lo es. Hay lecturas de texto y lecturas de video.

Los ejercicios en el Libro tienen un significado diferente al de los problemas. En un ejercicio ya se sabe del tipo de situación planteada y de que existe un procedimiento para resolverlo. Lo que se busca es que se utilice ese procedimiento y que, en lo posible, se desarrolle cierta soltura en el manejo de estas situaciones de manera que se adquiera rapidez y precisión en lo que se hace.

Las tareas se refieren a las actividades que los alumnos deberán realizar fuera del horario de clase. Buena parte de ellas consiste en el trabajo que deberán hacer con su libro de texto, pero también están las lecturas y la conclusión de alguna actividad que no se terminó en clase.

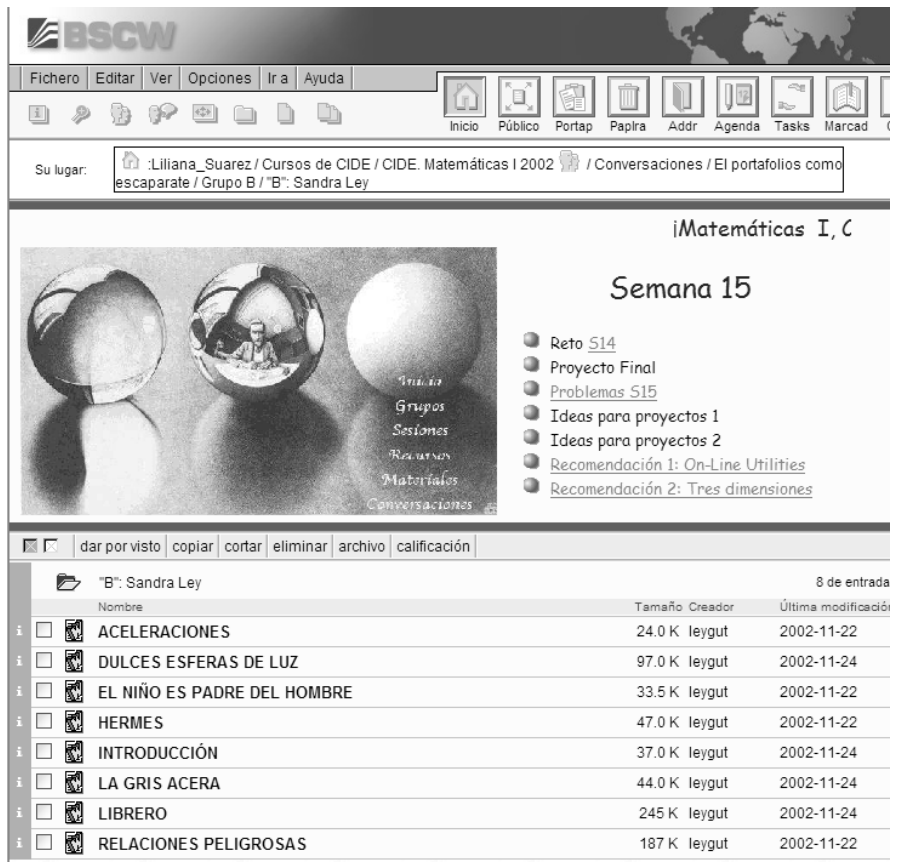

Fig. 3. Portafolios de un estudiante en la plataforma bscw. 
Liliana SuÁrez, Francisco Cordero, Patricio Daowz, Pedro Ortega, Alfonso Ramírez y José Torres

DE LOS PAQUETES DIDÁCTICOS HACIA UN REPOSITORIO DE OBJETOS DE APRENDIZAJE:

UN RETO EDUCATIVO EN MATEMÁTICAS. USO DE LAS GRÁFICAS, UN EJEMPLO

Las autoevaluaciones le permiten al estudiante conocer su comprensión y dominio de los temas tratados. El profesor conoce las soluciones de las mismas, y su actividad principal es señalar al alumno el momento adecuado de utilizarlas y destacar un aspecto que usualmente se olvida en las evaluaciones: identificar dónde se tienen deficiencias y, en consecuencia, se tiene que trabajar más.

De tal manera que se puede abordar la información de forma secuencial.

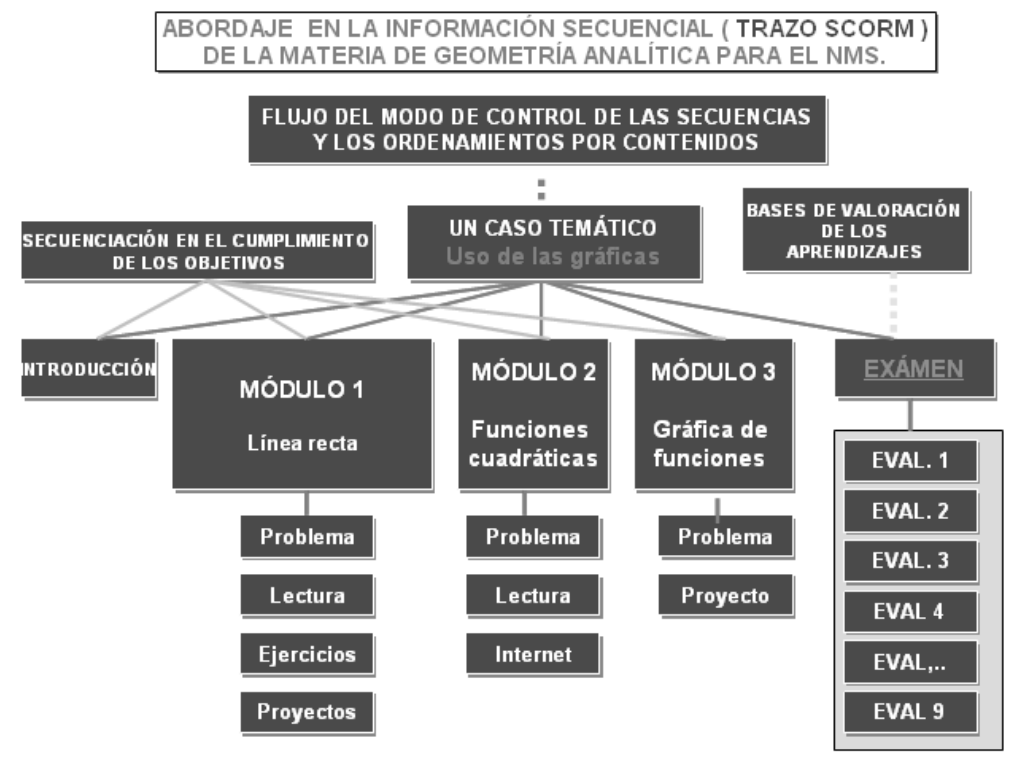

Fig. 4. Secuenciación de actividades de aprendizaje para el uso de las gráficas.

\section{La caracterización de las actividades}

En cada unidad se presentan actividades de aprendizaje para los alumnos. Estas actividades están presentadas por bloques de horas, suponiendo un total de 72 horas para el curso. El profesor debe revisar todas las actividades propuestas para cada unidad y planear explícitamente la secuencia que va a llevar a cabo con sus estudiantes, de manera que seleccione las actividades de la unidad, combinando distintos tipos para lograr los objetivos de aprendizaje. El profesor tendrá presente que se pueden identificar tres momentos importantes en el trabajo de los profesores: planear, instrumentar y evaluar cada actividad de aprendizaje de nuestros alumnos. 
Liliana Suárez, Francisco Cordero, Patricio Daowz, Pedro Ortega, Alfonso Ramírez y José Torres

DE LOS PAQUETES DIDÁCTICOS HACIA UN REPOSITORIO DE OBJETOS DE APRENDIZAJE:

UN RETO EDUCATIVO EN MATEMÁTICAS. USO DE LAS GRÁFICAS, UN EJEMPLO

Conviene recordar que es enriquecedor compartir con otros docentes las inquietudes y los resultados de las actividades de aprendizaje que realizó, y más enriquecedor todavía si planea y evalúa las actividades en equipo con otros colegas.

Para hacer la historia de las actividades de aprendizaje el profesor cuenta con el formato de caracterización de las actividades. La información que aporta, al caracterizarlas, sirve para que el profesor tome mejores decisiones en los distintos niveles que debe considerar cuando diseña una trayectoria para sus estudiantes.

Para conformar y caracterizar la red de actividades que comprende el paquete, se definieron diez características:

\begin{tabular}{|l|l|}
\hline 01. Experiencia de aprendizaje & 06.Producto \\
\hline 02. Modalidad de trabajo & 07.Referencias curriculares \\
\hline 03. Lugar de realización & 08.Representaciones \\
\hline 04. Herramientas tecnológicas & 09.Estrategias \\
\hline 05. Tiempo & 10.Evaluación \\
\hline
\end{tabular}

\section{Observaciones}

Con esta caracterización de las actividades de aprendizaje, se puede establecer explícitamente la vinculación que hay entre ellas desde perspectivas diferentes que se deben articular para organizar una sesión de clase. En el rubro de 'Referencias curriculares' se consideraron, además de los contenidos que marca el programa, algunos contenidos procedimentales y actitudinales, las competencias básicas del estudiante de bachillerato y los estándares 9-12 del NCTM. La complejidad del diseño y de la instrumentación de las actividades no se riñe con una consideración del tiempo disponible, que debe ser suficiente para que los estudiantes puedan realizar realmente las actividades, y de otros factores importantes como el nivel de desarrollo cognitivo de los estudiantes, sus ideas previas, sus expectativas y la pertinencia de los contenidos, que suelen variar para cada grupo de estudiantes en particular. Por el contrario, si el profesor dispone de más información se espera que la use para armonizar un trabajo que conduzca a un aprendizaje verdaderamente significativo para el estudiante.

Esta caracterización permita que los contenidos sean reutilizados con diferentes herramientas matemáticas en distintos contextos tecnológicos y curriculares. 
Liliana SuÁrez, Francisco Cordero, Patricio Daowz, Pedro Ortega, Alfonso Ramírez y José Torres

DE LOS PAQUETES DIDÁCTICOS HACIA UN REPOSITORIO DE OBJETOS DE APRENDIZAJE:

UN RETO EDUCATIVO EN MATEMÁTICAS. USO DE LAS GRÁFICAS, UN EJEMPLO

\section{Los ejemplos}

En el paquete se incluyen algunos ejemplos de los documentos que se consideran útiles para el trabajo del profesor. Se presenta el desarrollo de la solución que podemos esperar que produzcan los estudiantes del nivel medio superior y que llamamos 'de referencia', sin dejar de lado las variantes posibles. También se incluye un comentario de la actividad que se detiene en las distintas vías que puede seguir un estudiante, con la aplicación de las estrategias correspondientes, para avanzar en la solución de la actividad y describe la articulación de las representaciones. Apunta algunas sugerencias para la interacción con los estudiantes, en forma individual o en equipo, durante la realización de la actividad y para la discusión de las soluciones que se hace con todo el grupo. El comentario concluye con una ficha que resume los aspectos más importantes. Así se irán conformando historias de problemas, en particular, y de actividades, en general, que se robustecerán cada vez que las trabajemos en clase. Estas historias se harán más detalladas y útiles en la medida en que podamos elaborar los documentos que se describen en la sección siguiente. Esta labor la podremos emprender mejor aprovechando la red de interacción académica en Internet. En el anexo 1 puede verse un ejemplo en el que se incorporan dispositivos de transducción, sensores y calculadoras con poder de graficación, para el registro, el análisis y la interpretación de datos diversos en el salón de clases, en las experiencias de aprendizaje (Suárez, 2006).

\section{El trabajo del profesor}

En el paquete se presenta una forma de trabajo que toma en cuenta las características del quehacer docente mencionadas antes y, por tanto, se puede modificar o adaptar aprovechando la información que aporta. Cada profesor tiene su estilo de docencia, que se puede beneficiar de una práctica y una reflexión más sistemáticas, así como de las discusiones que se realicen alrededor de nuestras preocupaciones comunes. En nuestras Academias y en la red de interacción en Internet podemos ventilar nuestras inquietudes y dificultades y beneficiarnos de los comentarios y sugerencias de nuestros colegas.

Para utilizar las actividades en una sesión de clase, hay que hacer un plan, instrumentarlo y evaluarlo. Esta terna se repite en distintos niveles: la actividad, la clase, el tema, la unidad, el curso, el área, el ciclo, etc. Necesitamos desarrollar la habilidad de usar una especie de zoom que nos permita destacar los aspectos 
Liliana Suárez, Francisco Cordero, Patricio Daowz, Pedro Ortega, Alfonso Ramírez y José Torres

DE LOS PAQUETES DIDÁCTICOS HACIA UN REPOSITORIO DE OBJETOS DE APRENDIZAJE:

UN RETO EDUCATIVO EN MATEMÁTICAS. USO DE LAS GRÁFICAS, UN EJEMPLO

importantes que corresponden a cada nivel como el zoom lo hace con la escala. En cada acto de enseñanza, consideramos los objetivos de niveles distintos con los que se relaciona y la forma en que lo hace. Por ejemplo, si se trata de una experiencia necesaria pero que no genera un aprendizaje inmediato exigible, como es el caso de algunas de las líneas que apuntan al desarrollo de las habilidades intelectuales de orden superior, establecemos los lineamientos de interacción con los alumnos y los criterios de evaluación correspondientes, vinculándolos con otras experiencias de aprendizaje posteriores y haciendo inferencias explícitas sobre el desarrollo de la comprensión de los conceptos y procesos que se ponen en juego. Así mismo identificamos, desde una perspectiva sistémica, los factores que influyen en su práctica para establecer estrategias de acción, aun cuando la posibilidad de actuar sobre algunos factores sea muy escasa. En este sentido, es importante que nos veamos como parte de diferentes subsistemas y nos propongamos ampliar gradualmente nuestro campo de competencia y responsabilidad. El «zoom del profesor» se constituye así en una herramienta para, desde perspectivas distintas pero pertinentes, superar algunos callejones sin salida que parecen tales cuando sólo se atiende a la perspectiva del salón de clases.

A modo de ilustración se presenta cómo se puede planear, instrumentar y evaluar una sesión de resolución de problemas.

\section{La Planeación de una Sesión de Trabajo}

Aquí describimos una manera de organizar una sesión a partir de una actividad, que permite generar información sobre estos aspectos en cada instrumentación, conformando una 'historia del problema' o, en general, de la actividad de aprendizaje.

La fase de planeación requiere un análisis de la actividad desde un marco de referencia (Alarcón et al, 1996) y el registro por escrito de ese análisis. Esto le permitirá al profesor definir previamente no sólo la actividad que trabajará, cuál es el objetivo de la sesión y los tiempos disponibles, sino también cuáles son los obstáculos con los que se puede topar el alumno, cuáles van a ser sus actitudes ante los obstáculos, hasta donde debe llegar la sesión y en caso de no lograrlo qué hará para cumplir sus objetivos. Uno de los objetivos de la planeación es hacer explícitas nuestras expectativas. 
Liliana Suárez, Francisco Cordero, Patricio Daowz, Pedro Ortega, Alfonso Ramírez y José Torres
De los Paquetes didácticos hacia un repositorio de ObJetos de APrendizaje: UN RETO EDUCATIVO EN MATEMÁTICAS. USO DE LAS GRÁFICAS, UN EJEMPLO



Fig. 5. La planeación de un problema.

Por supuesto que lo que ocurrirá en la sesión de trabajo no puede estar completamente definido. Dentro del salón de clases el profesor toma decisiones constantemente con base en el marco de referencia que le brindan los documentos de la planeación y la información que va registrando durante la sesión. La planeación, entonces, debe ser flexible. Los documentos que concretarán nuestra planeación son:

Propósito de la actividad: que se manejará no únicamente desde la perspectiva de un contenido programático sino considerando las representaciones que articula (gráfica, aritmética-tabular, textual, algebraica, icónica, etc.), los aprendizajes que prepara, las categorías de resolución de problemas y los objetivos institucionales. El propósito de la actividad debe considerar que no todos los aprendizajes pueden ser inmediatos y que hay cuestiones que sólo se logran a largo plazo.

Recomendaciones durante la actividad: Cada uno de estos documentos está enfocado a los momentos que constituyen la sesión de trabajo, son una guía que nos permite dirigir la sesión hacia el objetivo establecido, sin desvirtuar la actividad.

a) Lineamientos para la interacción con los equipos: darán las pautas a seguir en la interacción del profesor con los alumnos mientras realizan la actividad (Suárez, 2006). La intervención de un profesor debe estar guiada por el ambiente, en el sentido de no invalidar el trabajo de los alumnos ni privarlos de la satisfacción de encontrar la solución por ellos mismos. 
Liliana Suárez, Francisco Cordero, Patricio Daowz, Pedro Ortega, Alfonso Ramírez y José Torres

DE LOS PAQUETES DIDÁCTICOS HACIA UN REPOSITORIO DE OBJETOS DE APRENDIZAJE:

UN RETO EDUCATIVO EN MATEMÁTICAS. USO DE LAS GRÁFICAS, UN EJEMPLO

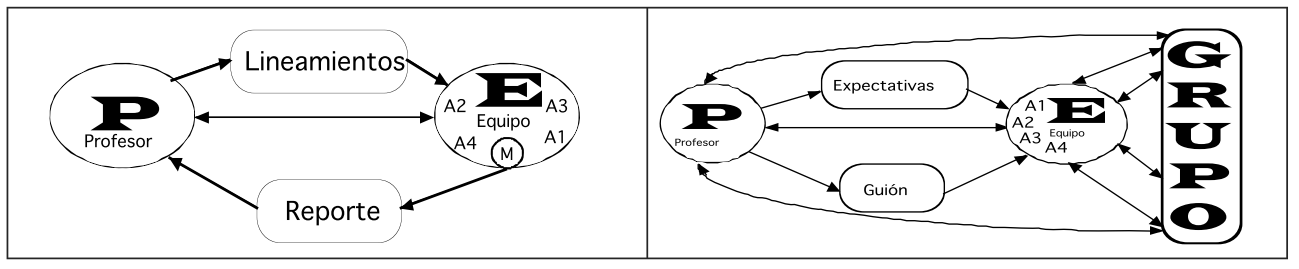

Figuras 6 y 7. La interacción del profesor con un equipo. Las interacciones durante la discusión del trabajo de un equipo.

b) Guión de la discusión: brinda un marco para la conducción de la discusión. Se consideran los posibles desarrollos de las soluciones y se establecen los lineamientos para la participación del profesor.

Recomendaciones para la evaluación de la actividad: La evaluación de la actividad debe considerar por lo menos:

a) Solución de referencia: Esta solución se elabora considerando los conocimientos que se ponen en juego durante la resolución del problema o la realización de la actividad.

b) Precepto de evaluación: Este documento contiene la descripción de los estándares de evaluación de un problema en particular. El precepto debe reflejar los principales aspectos del problema y aportar información útil para orientar el curso de las acciones del profesor y del estudiante ya sea para avanzar o profundizar en los contenidos que se pusieron en juego en el problema o para corregir las ideas erróneas que se hayan identificado.

\section{La Instrumentación}

La planeación debe tomar en cuenta los cursos diversos que pueden seguir la acción durante la instrumentación y sus posibles consecuencias en función de los propósitos de la sesión.

No es conveniente prodigar los comentarios ni las reformulaciones. Sin embargo, hay algunas intervenciones en las que se pueden solicitar aclaraciones, precisiones, explicaciones, justificaciones, cuando el profesor advierte indicios de perplejidad o incomodidad en el equipo o en el grupo que no logran formularse. La disyuntiva fundamental del profesor es decidir cuándo conviene detenerse para profundizar algún aspecto matemático. 
Liliana SuÁrez, Francisco Cordero, Patricio Daowz, Pedro Ortega, Alfonso Ramírez y José Torres

DE LOS PAQUETES DIDÁCTICOS HACIA UN REPOSITORIO DE OBJETOS DE APRENDIZAJE: UN RETO EDUCATIVO EN MATEMÁTICAS. USO DE LAS GRÁFICAS, UN EJEMPLO

Las intervenciones del profesor deben estar guiadas por los lineamientos para la interacción con los equipos y por el guión de la discusión de tal manera que no se vaya a desvirtuar la experiencia de aprendizaje que le corresponde disfrutar a los estudiantes con comentarios impacientes o irreflexivos. Hay un principio básico para que los documentos que concretan la planeación resulten útiles: antes de hablar, hay que escuchar.

\section{La Evaluación de la Actividad}

Después de realizada la actividad el profesor debe evaluar la efectividad y los resultados que se obtuvieron. No se trata sólo de la evaluación de los conocimientos, las habilidades, las actitudes y la transferencia del alumno. La evaluación de la actividad debe aportar información útil y confiable para mejorar el diseño de la actividad.

Además de la evaluación en los alumnos, se tiene la evaluación de la actividad y parte importante de ella es 'historiar el problema'. La idea es contrastar los análisis previo y posterior a la instrumentación para hacer un registro cada vez más robusto de las interacciones posibles, las formas de comprensión y el uso de las matemáticas que hacen los alumnos. Este contraste se puede complementar muy provechosamente con la investigación de los problemas y las condiciones en que se originaron los conceptos que se ponen en juego. Pero, puesto que nuestra perspectiva es la del profesor, y lo que necesariamente hace el profesor es trabajar con los alumnos, hemos optado por basarnos en nuestra experiencia y en la disposición de hacer explícitas nuestras expectativas para que, aun cuando nuestro primer análisis sea muy rudimentario, se vaya robusteciendo en las sucesivas puestas en escena, de tal manera que esta historia del problema se constituya en un saber propio del profesor generado en su práctica. Los registros audiovisuales brindan la oportunidad de aprovechar las ventajas de un análisis más detenido para incorporar sus resultados en las historias de los problemas. Las primeras versiones de la historia de una actividad pueden ser muy rudimentarias, e incluso contener sólo esbozos de algunos de los documentos que la integran, pero las contribuciones de los profesores las convierten rápidamente en un robusto conjunto de referencias que puede ser muy útil al profesor. Así se concreta una de las ideas principales de una organización profesional: la comunidad apoya sustancialmente el ejercicio profesional del individuo. 


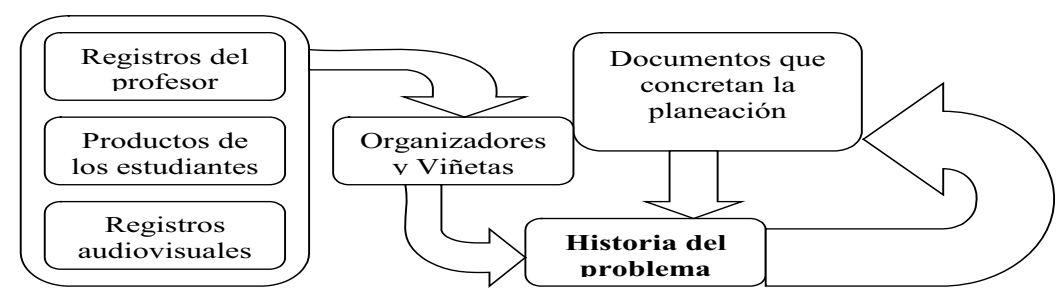

Fig. 8. La historia de un problema.

\section{Los productos del Proyecto}

En la actualidad se cuenta ya con los Paquetes Didácticos, para el profesor y para el estudiante, en versiones impresas y en Internet, de los cursos de Álgebra, para primer semestre, Geometría y Trigonometría, para segundo semestre, Geometría Analítica para tercero y diversos grados de desarrollo de los Paquetes correspondientes a los otros tres cursos del NMS.

Para la difusión de los paquetes entre los profesores del bachillerato del IPN se diseñaron y realizaron talleres sobre el 'Manejo del Paquete Didáctico de Álgebra'. El seguimiento y evaluación que se haga de los mismos permitirá hacer correcciones para la segunda edición.

\section{Hacia la constitución de un repositorio de objetos de aprendizaje}

Se ha descrito a grandes rasgos el proyecto de Paquetes Didácticos de Matemáticas, en el se presenta una propuesta para el desarrollo de los seis cursos de matemáticas del nivel medio superior, de acuerdo a planes de estudio vigentes, del Instituto Politécnico Nacional.

Los instrumentos para la caracterización de cada actividad de aprendizaje proporcionan una base para hacer una aproximación a la disección de cada una de ellas lo que permitirá evaluar su potencial de accesibilidad, interoperabilidad, durabilidad y reutilización. 
Liliana Suárez, Francisco Cordero, Patricio Daowz, Pedro Ortega, Alfonso Ramírez y José Torres

DE LOS PAQUETES DIDÁCTICOS HACIA UN REPOSITORIO DE OBJETOS DE APRENDIZAJE: UN RETO EDUCATIVO EN MATEMÁTICAS. USO DE LAS GRÁFICAS, UN EJEMPLO

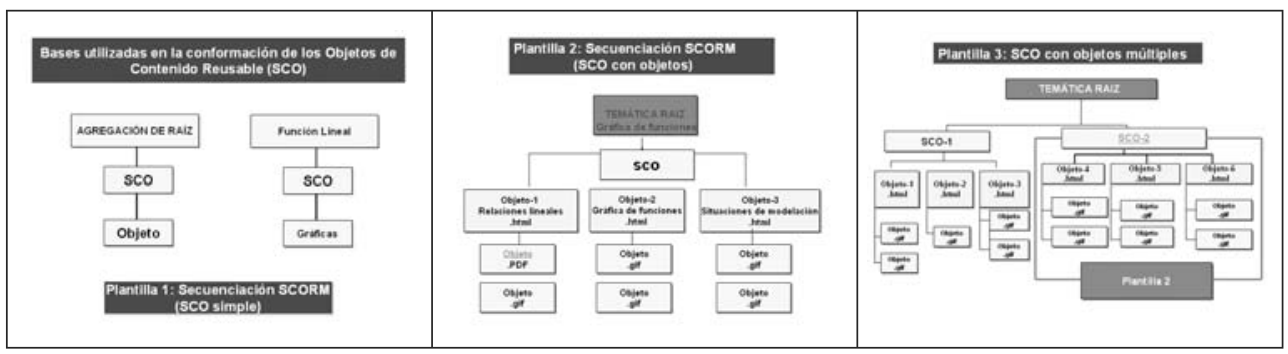

Fig. 9. Plantillas para la secuenciación de objetos de aprendizaje

La figura anterior proporciona un ejemplo de plantillas que pueden usarse para conformar el repositorio de objetos de aprendizaje para las secuencias de actividades de los Paquetes Didácticos de Matemáticas.

En este documento se han descrito los principales elementos de estos materiales que incluyen tanto herramientas tradicionales como herramientas innovadoras (discos compactos, sitios en Internet y Ambientes Virtuales de Aprendizaje administrados mediante las plataformas Blackboard y BSCW). Estos materiales han permitido lograr un mejor aprovechamiento por parte de los estudiantes, y constituyen los primeros pasos dados para que profesores y estudiantes del IPN conformen una Comunidad Virtual de Aprendizaje. El reto que sigue es integrar una metodología de desarrollo de contenidos digitales en la que se incorporen estudiantes y profesores de matemáticas del Instituto Politécnico Nacional.

\section{NOTAS}

$1 \quad$ Esta investigación fue apoyada por CONACYT con el proyecto Estudio de las gráficas de las funciones como prácticas institucionales. Una gestión escolar para el Nivel Superior. Clave No. 47045.

\section{REFERENCIAS BIBLIOGRÁFICAS}

AIM-NMS-IPN (2006) Comunidad virtual de la Academia Institucional de Matemáticas.

AIM-MNS-IPN (2005) Sitio en Internet del Paquete Didáctico de Geometría Analítica. [en línea]. Disponible en: http://www.te.ipn.mx/geometria/htm/ index.html. [consulta 19 de octubre de 2006].
AIM-NMS-IPN (2002). Comunidad Virtual para el Seguimiento de la Instrumentación del Paquete Didáctico de Matemáticas 1. [en línea]. Disponible en: $\quad$ http://bscw.fit.fraunhofer.de/ bscw/bscw.cgi/36478463 (se requiere autorizaciónlsuarez@ipn.mx). [consulta 19 de octubre de 2006]. 
Liliana Suárez, Francisco Cordero, Patricio Daowz, Pedro Ortega, Alfonso Ramírez y José Torres

DE LOS PAQUETES DIDÁCTICOS HACIA UN REPOSITORIO DE OBJETOS DE APRENDIZAJE:

UN RETO EDUCATIVO EN MATEMÁTICAS. USO DE LAS GRÁFICAS, UN EJEMPLO

Alarcón, J. et al. (1996). Un marco para el análisis de problemas. Memoria del Seminario 'Precálculo y resolución de problemas' realizado en el DMECINVESTAV-IPN.

Brenson-Lazan, G.(2001) Etapas de desarrollo y facilitación en una comunidad virtual de aprendizaje. [en línea]. Disponible en: http://amauta. org/DesarrolloComunidadVirtual.pdf [consulta 19 de octubre de 2006].

IPN (2004). Álgebra. Guía del estudiante. Instituto Politécnico Nacional. Obra completa 970-36-0178-2. Obra individual 970-36-0176-6. 2nda edición, México.

IPN (2006). Geometría Analítica. Libro del Profesor. IPN. ISBN: 970-36-0258-4.

IPN (2004). Álgebra. Guía del Profesor. Instituto Politécnico Nacional. ISBN: 970-18-6931-1.

IPN (2004). Materiales para la reforma. Publicaciones 01 a 19. [en línea]. Disponible en: http://www.mreforma. ipn.mx/ [consulta 9 de octubre de 2006]

Suárez, L. (2006) El uso de las gráficas en la modelación del cambio. Un estudio socioepistemológico para la modelación del cambio en un ambiente tecnológico. Memoria predoctoral no publicada. Departamento de Matemática Educativa, Cinvestav-IPN.

Suárez, L. (2000). El trabajo en equipo y la elaboración de reportes en un ambiente de resolución de problemas. Tesis de Maestría del DME-CINVESTAV-IPN.

Suárez, L.; Ortega, P.; Servin, Y.; Téllez, J.; Torres, J. L. (2005) Paquetes Didácticos de Matemáticas: Integración de la investigación y la innovación tecnológica. Extenso publicado en las Memoria de Virtual Educa. México, D. F. 2006. [en línea]. Disponible en: http://somi.cinstrum.unam.mx/ virtualeduca2005/resumenes/2005-0331456Matematicas VirtualEduca.doc [consulta 19 de octubre de 2005].

Torres, A.; Suárez, L.; Cordero, F. (2006). La modelación y las gráficas en situaciones de movimiento con tecnología. Extenso aceptado para su publicación en las Memorias del XXII Simposio Internacional de Computación en Educación IPN, México, 2006.

\section{PALABRAS CLAVE}

Objetos de aprendizaje, Reutilización, Redes de aprendizaje, Contenidos educativos digitales, Uso de las gráficas, Paquetes didácticos.

\section{KEY WORDS}

Digital Educational Contents, Graphics Use, Didactic Packages, Learning Objetcs, Reusability, Learning Networks. 
Liliana SuÁrez, Francisco Cordero, Patricio Daowz, Pedro Ortega, Alfonso Ramírez y José Torres

DE LOS PAQUETES DIDÁCTICOS HACIA UN REPOSITORIO DE OBJETOS DE APRENDIZAJE: UN RETO EDUCATIVO EN MATEMÁTICAS. USO DE LAS GRÁFICAS, UN EJEMPLO

\section{Anexo 1. Problemas resueltos (una guía didáctica para el profesor)}

Tomado de una puesta en escena didáctica de una tesis de maestría inscrita en el marco de la Matemática Educativa cuyo propósito es dar cuenta de los aprendizajes que logran los estudiantes del Nivel Medio Superior del Instituto Politécnico Nacional (IPN) al trabajar con un problema de una situación real de movimiento empleando tecnología. (Torres et al, 2006).

Problema

Epifanía

"Valentina llegó temprano a su clase de música. A punto estaba de sentarse cuando advirtió que había olvidado su cuaderno en su refugio predilecto: la siempre cómoda y acogedora biblioteca. No podía perderse el comienzo de la clase, así que fue a la biblioteca, cogió su cuaderno y regresó a su asiento, a tiempo para comenzar su, probablemente disfrutable, clase de música. Pero en el camino se encontró a su bienamado Juan y se detuvo a intercambiar algunas muestras de su muy auténtico cariño, lo que le llevó 4 minutos, pero de los largos, lo que la obligó a recuperar estos instantes, tan bien aprovechados, porque cuando salió del salón no previó la Epifanía”.

La biblioteca está en un punto diametralmente opuesto del salón de música en el patio circular, que tiene 500 metros de diámetro, de la escuela. Valentina tardó en total 9 minutos.

1) Construye una gráfica que describa los cambios de posición de Valentina en su trayecto de ida y vuelta con respecto al tiempo.

2) Todos hemos escuchado o hecho descripciones de objetos en movimiento, que incluyan expresiones como 'detenido', 'rápido', 'lento', 'más rápido', 'disminuyó su velocidad', 'más alejado', 'aceleró más', y muchas otras que seguramente te han asaltado la memoria.

Convengamos en que la velocidad de Valentina es positiva cuando se dirige a la biblioteca y negativa en sentido contrario.

Identifica en la gráfica intervalos en los que la velocidad sea negativa, positiva o nula, y describe las características de la gráfica, al igual que en el párrafo anterior, introduce matices en la descripción de la velocidad y anota las características correspondientes de la gráfica. 
Liliana Suárez, Francisco Cordero, Patricio Daowz, Pedro Ortega, Alfonso Ramírez y José Torres

DE LOS PAQUETES DIDÁCTICOS HACIA UN REPOSITORIO DE OBJETOS DE APRENDIZAJE:

UN RETO EDUCATIVO EN MATEMÁTICAS. USO DE LAS GRÁFICAS, UN EJEMPLO

\section{Solución y comentario didáctico}

Se considera que este problema sirve como una buena situación de aprendizaje ya que cumple en gran medida con las expectativas planteadas en lo que representa una caracterización de un buen problema (Suárez, 2000). Dicha caracterización es parte de los trabajos realizados por la AIM (IPN, 2002) puesto que sirve para identificar si un problema o actividad de aprendizaje tiene un potencial para que los estudiantes logren aprendizajes significativos.

Por lo que respecta al problema planteado podemos afirmar que:

- Es una experiencia de aprendizaje de resolución de problemas.

- La modalidad del trabajo se realiza en equipos de tres a cuatro estudiantes.

- El lugar de realización es en el salón de clases.

- Las herramientas utilizadas durante los trabajos son: Calculadora con poder de graficación, sensor de movimiento CBR, proyector de acetatos, pantalla líquida, cámara de video y fotográfica.

- El tiempo estimado para su realización es de tres horas.

- Son considerados como productos de la actividad los reportes de los estudiantes y registros audiovisuales de su trabajo.

El problema planteado cuenta con cuatro intervalos posibles de encuentro de Valentina con Juan [Ver Cuadro 1]: primero cuando se lo puede encontrar en el camino a la biblioteca, segunda cuando se lo encuentra de regreso al salón de clases, tercera cuando se lo encuentra al salir del salón de clases y cuarta cuando se lo encuentra en la biblioteca.

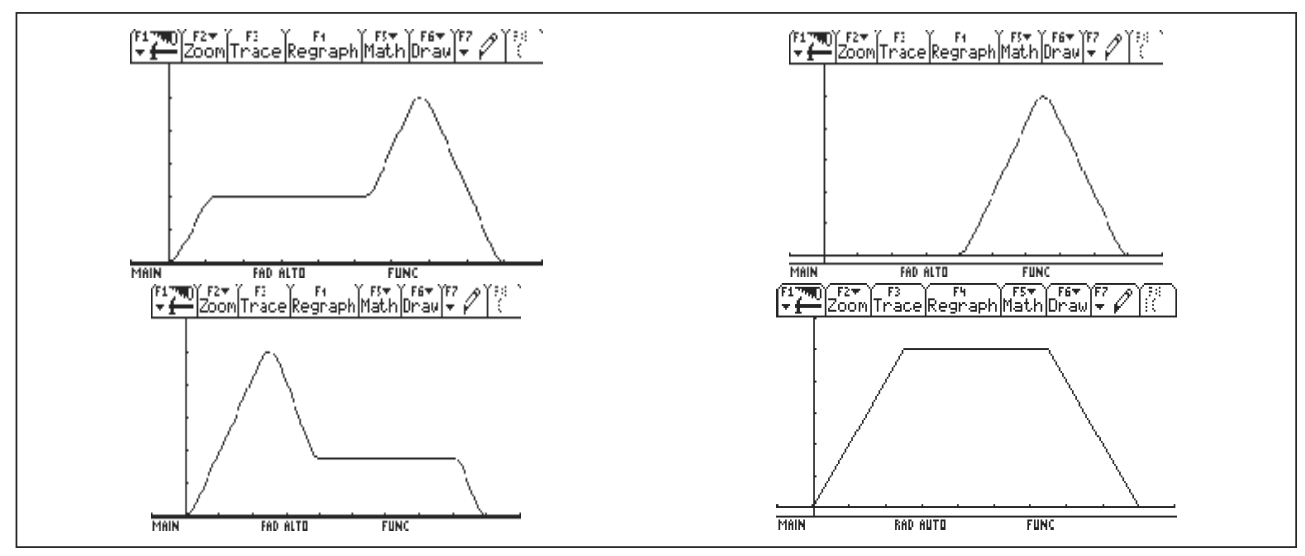

Cuadro 1 Cuatro posibilidades de encuentro 
Liliana Suárez, Francisco Cordero, Patricio Daowz, Pedro Ortega, Alfonso Ramírez y José Torres

DE LOS PAQUETES DIDÁCTICOS HACIA UN REPOSITORIO DE OBJETOS DE APRENDIZAJE:

UN RETO EDUCATIVO EN MATEMÁTICAS. USO DE LAS GRÁFICAS, UN EJEMPLO

A continuación se presenta el caso donde se lo encuentra en el camino hacia la biblioteca, para ello consideraremos primero rectas y después rectas y parábolas. La historia del problema, en las que se consideran las diferentes posiciones en las que se puede encontrar la persona, así como la identificación de manera en que ella realiza el movimiento (rápido, lento, más rápido, detenido), y el tipo de velocidad que se relaciona con cada cambio de dirección.

\section{Descripción del fenómeno de movimiento a través de rectas}

Como ya se mencionó el problema tiene varias posibilidades de solución, ya que el punto de encuentro no está determinado en el planteamiento del problema "Pero en el camino se encontró a su bienamado Juan y se detuvo a intercambiar algunas muestras de su muy auténtico cariño, lo que le llevó 4 minutos...”. El encuentro se puede dar en cualquiera de los tiempos entre $t=0$ y $t=5$, ya que si se lo encuentra después no puede permanecer con Juan cuatro minutos porque sabemos que regreso en $t=9$.

Tomando en cuenta que los desplazamientos que realiza Valentina son en línea recta, a continuación haremos cuando Valentina se encuentre a Juan antes de llegar a la biblioteca.

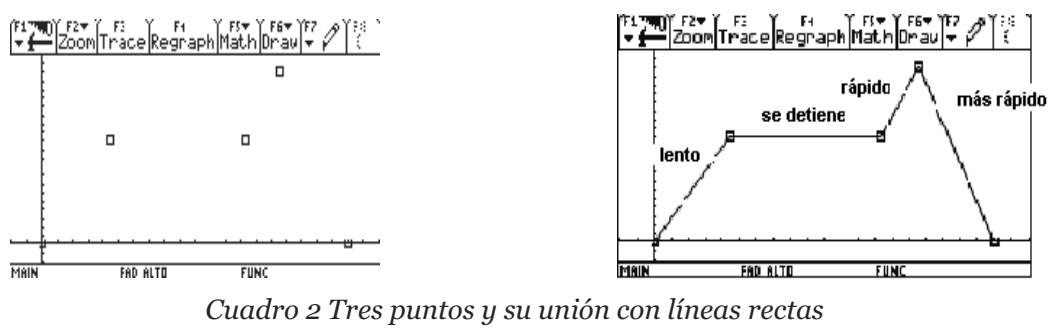

Como podemos observar en el Cuadro 2 hay cuatro intervalos de la posición de Valentina, el eje de las " $x$ " representa el tiempo que va de $0 \leq t \leq 9$ y el eje "y" la distancia recorrida por Valentina que va de $0 \leq d \leq 500$

a) Cuando Valentina sale del salón de clases y va en dirección a la biblioteca, aquí suponemos que Valentina recorre 300 metros en dos minutos, que es cuando se encuentra con Juan. Por lo que obtenemos la función de la recta que pasa por el origen, y por el punto $(2,300)$ cuya forma es:

$$
d=a t, \quad(0,0) y(2,300)
$$


Liliana Suárez, Francisco Cordero, Patricio Daowz, Pedro Ortega, Alfonso Ramírez y José Torres

DE LOS PAQUETES DIDÁCTICOS HACIA UN REPOSITORIO DE OBJETOS DE APRENDIZAJE:

UN RETO EDUCATIVO EN MATEMÁTICAS. USO DE LAS GRÁFICAS, UN EJEMPLO

Por lo que la función será: $\begin{aligned} & d=150 t \\ & 0 \leq t \leq 2\end{aligned}$

En consecuencia la velocidad de Valentina en este trayecto es de $150 \mathrm{~m} / \mathrm{min}$.

b) Después del recorrido anterior, y como ya dijimos Valentina se encuentra con Juan a los 300 metros, y ahí pierde cuatro minutos, por lo que no hay desplazamiento, la función de dicha recta que pasa por los puntos $(2,300)$ y $(6,300)$ es de la forma:

$d=a, \quad(2,300) y(6,300)$

Por lo que la función es: $\begin{aligned} & d=300 \\ & 2<t \leq 6\end{aligned}$

Como podemos observar la velocidad de Valentina en este lapso de tiempo es nula.

c) Pasados los cuatro minutos que se entretiene Valentina con Juan, vuelve a emprender el recorrido hacia la biblioteca, el cual le lleva un minuto en recorrer 200 metros que le faltaban para llegar a la biblioteca. La forma de la función de la recta de dicho recorrido, es de la forma:

$d=a t+b, \quad(6,300) y(7,500)$

La función será: $\begin{aligned} & d=200 t-900 \\ & 6<t \leq 7\end{aligned}$

Aquí la velocidad es de $200 \mathrm{~m} / \mathrm{min}$.

d) Finalmente Valentina tiene que recuperar el tiempo perdido, por lo que tiene que recorrer los 500 metros en dos minutos, para llegar a tiempo a su clase. La forma de la función de este último recorrido es el siguiente:

$d=-a x+b, \quad(7,500)$ y $(9,0)$

Dicha función es: $\begin{aligned} & d=-250 t+2250 \\ & 7<t \leq 9\end{aligned}$ 
Liliana Suárez, Francisco Cordero, Patricio Daowz, Pedro Ortega, Alfonso Ramírez y José Torres DE LOS PAQUETES DIDÁCTICOS HACIA UN REPOSITORIO DE OBJETOS DE APRENDIZAJE:

UN RETO EDUCATIVO EN MATEMÁTICAS. USO DE LAS GRÁFICAS, UN EJEMPLO

En este trayecto la velocidad de Valentina es de $-250 \mathrm{~m} / \mathrm{min}$. Resulta ser negativa porque ella ya va de regreso al salón de clases.

La función resultante de todo su trayecto es una función a trozos que podemos expresar de la siguiente manera:

$$
d=\left[\begin{array}{ll}
150 t, & (0 \leq t \leq 2) \\
300, & (2<t \leq 6) \\
200 t-900, & (6<t \leq 7) \\
-250 t+2250, & (7<t \leq 9)
\end{array}\right]
$$

Usando la calculadora graficadora podemos escribir dicha función, y ver la gráfica correspondiente. [Ver Cuadro III.3]

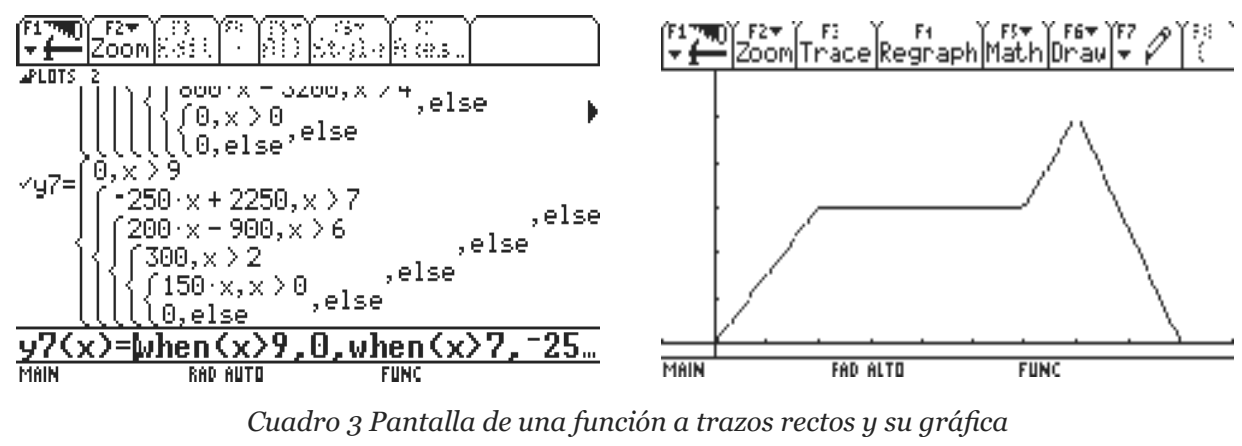


Liliana Suárez, Francisco Cordero, Patricio Daowz, Pedro Ortega, Alfonso Ramírez y José Torres

DE LOS PAQUETES DIDÁCTICOS HACIA UN REPOSITORIO DE OBJETOS DE APRENDIZAJE:

UN RETO EDUCATIVO EN MATEMÁTICAS. USO DE LAS GRÁFICAS, UN EJEMPLO

En cuanto a la velocidad y como ya se ha determinado durante los dos primeros minutos la velocidad es constante y de $150 \mathrm{~m} / \mathrm{min}$. su función entonces será:

$V=150$

Cuando se detiene a platicar con Juan su velocidad es igual a cero,

por lo que su función será:

$0 \leq t \leq 2$

$v=0$

$2<t \leq 6$

Pasados los cuatro minutos que estuvo con Juan continúa su camino hacia la biblioteca por lo que aumenta su velocidad, nuevamente se hace constante, y es de $200 \mathrm{~m}$ / $\mathrm{min}$. La función entonces sería:

Finalmente regresa al salón de clases por lo que recorre 500 metros en dos minutos y aquí su velocidad es de $-250 \mathrm{~m} / \mathrm{min}$. (es negativa porque va de regreso) la función es la siguiente:

La función resultante de la velocidad es una función a trozos que podemos escribir de la siguiente manera:

En el cuadro 4 (i) se muestra la gráfica de la velocidad, y en (ii) el contraste entre las gráficas de la posición y la de la velocidad.

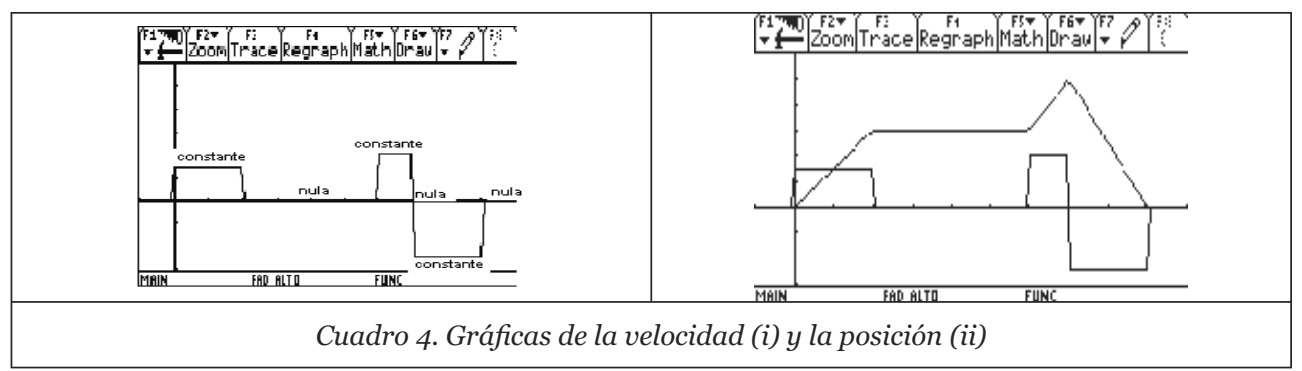

Descripción del fenómeno de movimiento a través de las gráficas de la simulación

En seguida se presentan las gráficas de la posición de Valentina [Cuadro III.8 (i)] y de la velocidad [Cuadro III.8 (ii)], que se obtuvieron después de hacer varios ensayos con el sensor y la calculadora graficadora, y en el Cuadro III.9 se presenta el contraste de las dos gráficas. 
Liliana Suárez, Francisco Cordero, Patricio Daowz, Pedro Ortega, Alfonso Ramírez y José Torres

DE LOS PAQUETES DIDÁCTICOS HACIA UN REPOSITORIO DE OBJETOS DE APRENDIZAJE:

UN RETO EDUCATIVO EN MATEMÁTICAS. USO DE LAS GRÁFICAS, UN EJEMPLO
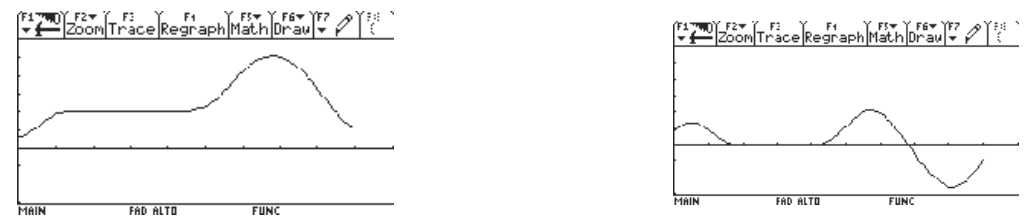

Cuadro 5. Gráficas de la posición (i) y la velocidad (ii) con simulación

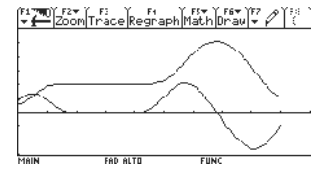

Cuadro 6. Gráficas simultáneas

Caracterización de la Actividad de Aprendizaje

Formato para la clasificación de problemas

\begin{tabular}{|ll|l|}
\hline \multicolumn{1}{|c|}{ Título } & \multicolumn{1}{c|}{ Epifanía } \\
\hline $\begin{array}{l}\text { 1. } \\
\text { Experiencia de } \\
\text { aprendizaje }\end{array}$ & 1.1 Resolución de problemas. \\
\hline 2. & Modalidad de trabajo & 2.2 Equipo; 2.3 Grupo. \\
\hline 3. & Lugar de realización & 3.1 Salón de clases. \\
\hline 4. & $\begin{array}{l}\text { Herramientas } \\
\text { tecnológicas }\end{array}$ & $\begin{array}{l}\text { 4.4 Calculadora con poder de graficación y sensores de } \\
\text { movimiento. }\end{array}$ \\
\hline & $\begin{array}{l}\text { 120 minutos (se puede trabajar en 2 sesiones de } \\
\text { 6o minutos cada una, la segunda sesión se puede } \\
\text { utilizar para aclarar conceptos y obtener una gráfica } \\
\text { más detallada, construida ya a partir de la función } \\
\text { algebraica, que la obtenida durante la primera parte } \\
\text { del trabajo con el problema). }\end{array}$ \\
\hline 5. Tiempo & 6.1 Reporte de RP. \\
\hline 6. & Producto & \\
\hline
\end{tabular}


Liliana Suárez, Francisco Cordero, Patricio Daowz, Pedro Ortega, Alfonso Ramírez y José Torres

DE LOS PAQUETES DIDÁCTICOS HACIA UN REPOSITORIO DE OBJETOS DE APRENDIZAJE:

UN RETO EDUCATIVO EN MATEMÁTICAS. USO DE LAS GRÁFICAS, UN EJEMPLO

\begin{tabular}{|c|c|}
\hline $\begin{array}{l}\text { 7. Referencias } \\
\text { curriculares }\end{array}$ & $\begin{array}{l}\text { 1.1 Contenidos } \\
\text { 1.1.1 Conceptuales (1.1); (2.1); (4.1, 4.2, 4.3) } \\
\text { 1.1.2 Procedimentales p1, p2, p3, p4, p5, p6, p8, p10, } \\
\text { p13, p15. } \\
\text { 1.1.3 Actitudinales a1, a2, a3, a6, a7, a9, a12, a13 } \\
\text { 1.2 Competencias básicas del estudiante de bachillerato } \\
\text { cb1, cb2, cb3, cb5, cb7 } \\
\text { 1.3 Estándares 2000 del NCTM 4.1.1, 6.2, 6.3, 6.4, 7.1, } \\
\text { 8.4, 10.1, 10.2. }\end{array}$ \\
\hline 8. Representaciones & $\begin{array}{l}\text { 8.1 Textual - 8.2 Tabular - 8.3 Gráfica - 8.1 Textual } \\
\text { - 8.3 Gráfica - 8.4 Algebráica. }\end{array}$ \\
\hline 9. Estrategias & $\begin{array}{l}\text { Toma una instantánea } \\
\text { Organiza la información en una tabla } \\
\text { Haz una gráfica } \\
\text { Usa una notación adecuada } \\
\text { Cambia el registro de representación } \\
\text { Trata casos particulares } \\
\text { Utiliza "La Lupa" }\end{array}$ \\
\hline 10. Evaluación & $\begin{array}{l}\text { Evaluación del reporte. } \\
\text { Evaluación de la presentación. }\end{array}$ \\
\hline Observaciones & $\begin{array}{l}\text { El problema debería tratarse, por lo menos, en } \\
\text { dos ocasiones. La primera se puede centrar en la } \\
\text { interpretación textual y de los significados de la } \\
\text { primera y segunda derivada, llegando a un esbozo de } \\
\text { la gráfica. En la segunda, se pondría especial atención } \\
\text { en la representación gráfica y en encontrar la ecuación } \\
\text { de la función. Discutiendo también la manera en } \\
\text { que se relacionan la primera y segunda derivadas de } \\
\text { una función con las características de la función y su } \\
\text { gráfica. Si se tuviera la posibilidad de retomar el tema } \\
\text { posteriormente, la discusión podría profundizar en } \\
\text { los aspectos que se hayan mencionado en las sesiones } \\
\text { anteriores, aprovechando las preguntas nuevas que se } \\
\text { incluyen al final del comentario. }\end{array}$ \\
\hline
\end{tabular}

En resumen, algunos aspectos del problema que conviene atender durante el trabajo de los equipos y retomar en la discusión con todo el grupo son: 
Liliana SuÁrez, Francisco Cordero, Patricio Daowz, Pedro Ortega, Alfonso Ramírez y José Torres

DE LOS PAQUETES DIDÁCTICOS HACIA UN REPOSITORIO DE OBJETOS DE APRENDIZAJE:

UN RETO EDUCATIVO EN MATEMÁTICAS. USO DE LAS GRÁFICAS, UN EJEMPLO

\begin{tabular}{|l|l|l|}
\hline Modelación & $\begin{array}{l}\text { Trascripción de situaciones problemáticas a un lenguaje } \\
\text { algebraico, utilizando las técnicas matemáticas apropiadas } \\
\text { para resolverlas y dar una interpretación, ajustada al } \\
\text { contexto, de las soluciones obtenidas. }\end{array}$ \\
\hline $\begin{array}{l}\text { Representación } \\
\text { gráfica }\end{array}$ & $\begin{array}{l}\text { Lectura local } \\
\text { Obtener la } \\
\text { gráfica a partir } \\
\text { de la descripción } \\
\text { de primeras } \\
\text { y segundas } \\
\text { derivadas. }\end{array}$ & $\begin{array}{l}\text { Intervalos de crecimiento, } \\
\text { decrecimiento, concavidad, mínimos o } \\
\text { máximos relativos. } \\
\text { Gráficas de la primera y segunda } \\
\text { derivada de una función, relacionada } \\
\text { con la gráfica de la función. }\end{array}$ \\
\hline & $\begin{array}{l}\text { Textual a organización tabular de la la } \\
\text { información. } \\
\text { Textual a gráfica. } \\
\text { Textual a algebraica. } \\
\text { Tabular a gráfica. } \\
\text { Gráfica a textual. } \\
\text { Gráfica a algebraica. } \\
\text { Algebraica a gráfica. }\end{array}$ \\
\hline $\begin{array}{l}\text { Función } \\
\text { Segunda derivada }\end{array}$ & $\begin{array}{l}\text { Representaciones } \\
\text { Primera derivada como criterio para definir la concavidad } \\
\text { Segunda derivada como criterio para definir la concavidad } \\
\text { de la gráfica de una función. } \\
\text { Segunda derivada como criterio para definir un máximo o } \\
\text { mínimo relativo. }\end{array}$ \\
\hline $\begin{array}{l}\text { Sistemas de } \\
\text { ecuaciones }\end{array}$ & $\begin{array}{l}\text { Reprener el sistema de ecuaciones, } \\
\text { resultados. } \\
\text { a partir de la información de las } \\
\text { primeras y segundas derivadas. } \\
\text { Identificar el polinomio apropiado } \\
\text { para generar el sistema de ecuaciones. }\end{array}$ \\
\hline Cambitmo e interpretación de los \\
\hline
\end{tabular}


Liliana Suárez, Francisco Cordero, Patricio Daowz, Pedro Ortega, Alfonso Ramírez y José Torres

DE LOS PAQUETES DIDÁCTICOS HACIA UN REPOSITORIO DE OBJETOS DE APRENDIZAJE:

UN RETO EDUCATIVO EN MATEMÁTICAS. USO DE LAS GRÁFICAS, UN EJEMPLO

\title{
PERFIL ACADÉMICO DE LOS AUTORES
}

Liliana Suárez Téllez actualmente desarrolla una investigación doctoral sobre el uso de las gráficas en la modelación del cambio en el Departamento de Matemática Educativa del Centro de Investigación y de Estudios Avanzados del IPN. Ha diseñado y participado en Programas de Formación Docente en matemáticas en el IPN y en algunos estados de la República Mexicana y el extranjero. Fue responsable del Portal de la Academia Institucional de Matemáticas: http://www.comunidades.ipn.mx/ aim desde su creación hasta 2005.

Dirección postal:

\author{
Liliana Suárez Téllez \\ Manuel González 42, Edificio Baja California “D”, 308 \\ Unidad habitacional Tlatelolco, México, D. F. 0690o, \\ México \\ Teléfono +5557820880 \\ Correo electrónico: 1suarez@ipn.mx
}

Francisco Cordero Osorio. Doctor en Ciencias, especialidad Matemática Educativa del Cinvestav-IPN y Postdoctorado en Purdue University, Estados Unidos. Es Miembro Regular de la Academia Mexicana de Ciencias, del Comité Latinoamericano de Matemática Educativa y de la Research in Undergraduate Mathematics Education Community. Ha publicado artículos en revistas internacionales y ha dictado conferencias en Universidades de países de Latinoamérica y de Estados Unidos, Francia, España y Turquía. Desarrolla la línea de investigación: Pensamiento Matemático Avanzado, en la aproximación socioepistemológica.

Dirección postal:

\author{
Dr. Francisco Cordero Osorio. \\ Departamento de Matemática Educativa del Cinvestav-IPN \\ Av. Instituto Politécnico Nacional 2508 \\ Col. San Pedro Zacatenco, 07360 México, D. F. \\ México \\ Correo electrónico: fcordero@cinvestav.mx
}

Patricio Daowz Ruiz. Licenciado en filosofía por la Universidad Nacional Autónoma de México. Maestría en filosofía por la Universidad Nacional Autónoma de México. Doctorado en planeación educativa con orientación a sistemas por la Universidad de Paris (Vincennes). Actualmente es subdirector de Modelos Educativos con Tecnología del Centro de Tecnología Educativa del IPN.

Dirección postal:

Dr. Patricio Daowz.

Correo electrónico: pdaowzr@ipn.mx 


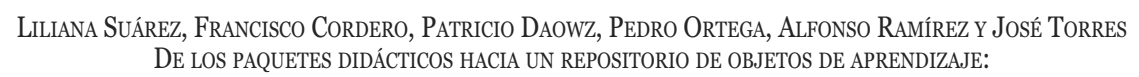
UN RETO EDUCATIVO EN MATEMÁTICAS. USO DE LAS GRÁFICAS, UN EJEMPLO

Pedro Ortega Cuenca es miembro fundador de la Academia Institucional de Matemáticas (AIM-NMS-IPN) y asesor desde su fundación en 1995 hasta 2005. Coordinador del proyecto 'Paquetes Didácticos de Matemáticas', proyecto cuyo propósito fue el diseño de materiales educativos para los seis cursos de Matemáticas de acuerdo a los planes de estudio del IPN de 1994 en formato impreso, disco compacto y sitios en Internet y plataformas educativas.

Dirección postal:

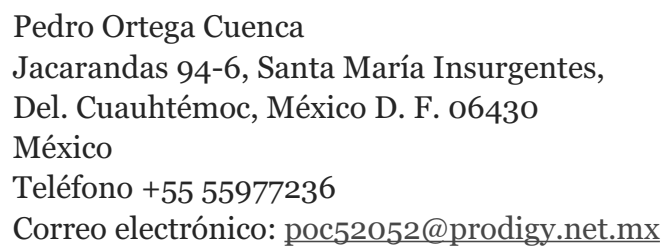

Alfonso Ramírez Ortega. Maestro en ciencias con especialidad en Matemática Educativa por el Centro de Investigación y Estudios Avanzados (CINVESTAV) del Instituto Politécnico Nacional (IPN). Acualmente es director del Centro de Tecnología Educativa del IPN.

Dirección postal:

M. en C. Alfonso Ramírez Ortega

Correo electrónico: aramireo@ipn.mx

José Luis Torres Guerrero es profesor de bachillerato en el Instituto Politécnico Nacional. Tiene grado de Maestría en Ciencias en la especialidad de Matemática Educativa. Formó parte de la Academia Institucional de Matemáticas del Nivel Medio Superior del IPN (1994-2005) y participó en proyectos de la misma, por ejemplo en la elaboración de Paquetes Didácticos de Matemáticas y en el ciclo de videoconferencias "Repensar las Matemáticas". Ha participado en congresos presentando ponencias y como tallerista.

Dirección postal:

José Luis Torres Guerrero

Correo electrónico: jeluistg@yahoo.com.mx

Fecha de entrega: 06.10.06

Fecha de aceptación: 19.12.06 